\title{
Bilingual Education in Sri Lankan Schools: An Analysis of Difficulties in Speaking English Experienced By Students in Selected Schools in Sri Lanka
}

\author{
M.D.N.M.U. MAHAWATTHA \\ Sabaragamuwa University of Sri Lanka \\ utthara.mahawattha@gmail.com
}

\begin{abstract}
Bilingual Education was introduced to Sri Lanka in 2001, initially through Amity School Programs. In this Bilingual Program, English is the medium of instruction in selected subjects from grade 6 to grade 11. The students of Bilingual Program are expected to be competent both in First Language- L1 (Sinhala/Tamil) and Target Language (English). However, the students find it difficult to express themselves in English especially in academic performances. The research findings of NIE, 2007, too, correspond with the relevant observation. I adapted the descriptive method to collect data by giving questionnaires to students in the sample of 60 students and 20 teachers including English teachers, non - English teachers who work in the Bilingual Program and teachers who work in Activity Based Oral English (ABOE) Program in primary education. An analysis of collected data suggests a $65 \%$ of the sample is not expressive themselves in English, especially in academic performances. This revelation is important because it helps to anticipate the propositions find in Bilingual Education and to take necessary steps to guide its move. In this paper, I try to interpret the difficulties in speaking in English faced by students in the Bilingual Education Program with the help of theoretical explanation.
\end{abstract}

Key Words: Bilingual Education, English, First Language - L1, Target Language, academic performances

\section{Introduction}

Bilingualism is the mastery of two languages. The regular use of two languages is the distinguished characteristic of a bilingual. Bilingualism may occur due to many reasons. Often people of minority groups become bilingual since they are 'forced' to learn the language of the majority in parallel to their first language (L1). At the same time, when a link language is widely used in a country apart from the state language, Bilingualism may occur. Definitions of Bilingualism cover a very broad spectrum of linguistic abilities. Children who are exposed to more than one language in early childhood are sometimes referred to as "simultaneous bilinguals". Those who begin to learn a second language later are referred to as "sequential bilinguals" (Lightbown and Spada, 1991). Number of researches has been indulged in speculation about the consequences of Bilingualism for children's cognitive development, linguistic processing and metalinguistic abilities. However, this paper focuses on sequential bilinguals and the difficulties in speaking in English faced by them in their Bilingual Education. To better understand this, it is important to recognize the spectrum of Bilingual Education and what Bilingual Education is in its broader perspective. Bilingual Education is an educational program, which has an involvement of two or more languages in varying degrees. Defined broadly, it can mean any use of two languages in school - by teachers or students or both - for a variety of social and pedagogical purposes. Bilingual Education has been practiced in many forms, in many countries. The objectives of Bilingual Education may vary from country to country depending on its cultural, social, economic and educational needs. The spectrum of Bilingual Education is very broad but three common types of Bilingual Education Programs can be identified as Transitional, Developmental and Immersion, depending on the instructional models used. In Sri Lanka, it is the Developmental Bilingual Program that was implemented in 2001, through Amity school Programs. In terms of Bilingual Education in Sri Lankan context, proposals of the National Education Commission-2003, state their standpoint under Bilingual Education Policy as follows:

Secondary Education: Bilingualism should be promoted by using English as the medium of instruction in selected subjects such as Mathematics, Science and Technology including Computer Literacy, Social Sciences in secondary grades, year by year, from Grade 6, depending on the availability of teachers. It is expected that 
students will reach acceptable level of proficiency in English at the end of junior secondary education without jettisoning Sinhala and Tamil which will continue to be the medium of instruction in selected subjects." ((Bilingual Education, Teacher Developmental Manual, 2007). Thus, Bilingual Education is implemented in Sri Lankan schools as suggested and the first batch who has studied under Bilingual Program has already faced their G.C.E. (O/L) in 2007. "Initially, the English medium in selected subjects were made available in National Schools and in schools identified as provincial centers of Excellence to which scholarship holders are to be sent, so that at least one or two schools in each administrative division could teach the selected subjects in English as early as possible" (Bilingual Education, Teacher Developmental Manual, 2007). However, by now, a number of schools are there all over the country, which conducts Bilingual Education Programs. There are different individuals in this program from different socio economic and cultural backgrounds. As a result, their cognitive capacities are highly influenced by these different environments. In a situation like Bilingual Education in Sri Lanka, the diversity of co-learners is higher than that of a normal classroom due to the diversity of language experiences of each individual student. Yet, many surveys (including my findings) have found out that the students of Bilingual Program face difficulties in speaking in English in their academic performances. Although this research focuses only on difficulties in speaking in English, it is important to remember that these four skills are more or less interrelated to each other. There are several reasons to focus this research on speaking. One reason is the 'negligence' of speaking in the second language classroom. The general objective of teaching - learning process in a language classroom focuses on to develop the four skills of students. Obviously, the four skills are equally important and they are integrated with each other. No skill can be treated in isolation. Speaking is highly related to listening than to reading and writing since those two skills are reciprocal. In second language teaching - learning process, often, speaking is less focused. There may be different reasons for this situation. However, unlike reading and writing, the role of speaking and listening is less than clear.

There is a considerable amount of guidance relating to the teaching of reading and writing but as far as speaking and listening is concerned, teachers may not be sure where to turn in order to be sufficiently informed or in control. Thus, speaking and listening remains an aspect of the second language teaching curriculum that is less rigorously and systematically, taught and assessed. Even in a bilingual classroom, students access literacy through oracy. Teachers are expected to use L2 when they teach selected subjects in English medium, especially when students reach Grade 9 - after completing three years in their Bilingual Program. Yet, when we think about second language learning, it is often observed that speaking is the least acquiring skill. Thus, it is important to assess speaking in Bilingual Program to find out how it affects bilingual learners' language development. I, as an English academic have observed that there is an allegation often leveled by teachers who work in the Bilingual Education Program that bilingual students cannot be as expressive as monolingual students in academic performances might be because they are not 'fluent' in English. This allegation is one of the reasons which sparked off this study, since I, as an English academic, wanted to find out if this as truly the case, and if so, what measures should be taken to remedy the situation. Therefore, this paper is based on the hypothesis that lack of 'fluency' in English is the reason for bilingual students' lack of expressions, especially in academic performances. In the discussion on results and findings in this paper, I shall examine if this hypothesis would be valid. The two research questions that I would like to answer through this research would be,

- Why do the students of Bilingual Education Program find difficulties in speaking in English?

- What is the place given to learning the L1 in order to develop the L2?

Thus, the purpose of this paper is to assist readers in clearly defining and articulating a personal and professional statement on difficulties in speaking in English faced by grade nine students in Bilingual Education Program in selected schools in Ratnapura district. With these objectives in mind, a decision was made to collect data from students themselves, who decide on their fluency. A total of 60 students and 20 teachers were interviewed and the findings of this research are based on the information provided by them. The students provided data in the form of responses to questionnaires. It is relevant to state that of these 60 students, a considerable number of students were of National Schools. The rest was from a "Navodya" school. A secondary objective was the collection of data from the teachers who work in the Bilingual Program and primary teachers of grades 1 and 2 who are supposed to deal with Activity Based Oral English. This study will support teachers in addressing many of the questions about effective teaching strategies in speaking in 
English for students in Bilingual Education Programs and challenges certain prejudices on Bilingual Education Program that may be posed by administrators, parents, community members and colleagues.

\section{Literature Review}

The introduction laid the basis in dealing with the question of difficulties in speaking in English faced by grade nine students in Bilingual Education Program, on which the proficiency in English is seen as a necessary characteristic to be excelled in Bilingual Education Program. With the advent of certain neuropsychological, psycholinguistic and social and psychological theories gaining recognition and popularity, many researchers have discussed the difficulties in L2 competency, by students in Bilingual Education Program where there have been arguments, theories and ideas either validating or refuting this particular issue. These theories, combined with awareness and education resulted in many countries striving to change the role of English education, where they continually asserted themselves in methods, which ranged from transitional to immersion and have now confronted the authority, the importance of First Language (L1) in Second Language (L2) learning, which has been long ignored. One can see the gradual development in Bilingual Education in Sri Lanka with more students coming in to bilingual mode, which was highly confined only for very few students at the beginning. I will be presenting and discussing the difficulties in speaking in English faced by grade nine students in Bilingual Education Program in selected schools in Ratnapura district, by first looking at the importance of L1 in L2 learning and how it has been discussed by researchers in their work. The importance of L1 in L2 learning is one of the main elements that has stratified and ranked the theoretical aspects and research findings related to Bilingual Education a great division among theorists. The norm that has been prevailing in the society from the past is that "the best medium for teaching a child is his mother tongue" (UNESCO, 1953, 11). Tracing the history of Bilingual Education, Cummins cites Darcy, 1953, Pearl, and Lambert, 1962, to view the prevailing attitudes in the past as follows: "A frequent finding was that bilingual children performed poorly on the verbal parts of intelligence tests as well as on academic tasks and several investigators argued that bilingualism itself was a cause of "mental confusion" and "language handicaps" (Cummins, 1979).

Further he points out that "the most coherent theoretical statement" which comprehended the 'negative' effect of L2 on L1 was McNamara's (1966) "balance effect" hypothesis which suggested that "a bilingual child paid for his L2 skills by a decrease in L1 skills" (Cummins, 1979, p. 223). Apart from that, the "linguistic mismatch" hypothesis, which suggested that mismatch between the language of the home and the language of the school leads to academic retardation, had aggravated the situation. Although these attitudes were intrigued several decades ago, NIE research which has taken place in 2007 shows that still there are "different myths" about the 'negative' effects of L2 on L1 (Bilingual Education, Teacher Developmental Manual, 2007). However, a large number of recent studies done by Baker (2000), Cummins (2000) and Kangas (2000) have shown that Bilingualism can positively influence both cognitive and linguistic development of children. Research done by Jim Cummins, of the Ontario Institute for Studies in Education at the University of Toronto, supports a basic tenet of Bilingual Education: Children's L1 should be well developed to get their academic and linguistic performance of L2 maximized. Cummins's Developmental Interdependence Theory suggests that successful growth in a second language is dependent upon a well- developed L1. This view is quite applicable to Sri Lankan Bilingual Education Program because, this bilingual mode is initially introduced considering different socio-political, economic, cultural and cognitive needs of the country. Bilingual Education in Sri Lanka is a long-term plan to preserve the language, culture and the heritage of the nation, while giving importance to a global language. Hence, in Sri Lankan context, dominant language is intended to remain dominant while the state of the L2 as weak L2 or as a foreign language is to be developed. As discussed in the introduction, and based on the NIE research findings it is obvious that students in the bilingual mode find difficulties in L2 competency and they come across difficulties in academic performance.

Cummins addresses the lack of competency in L2 in terms of bilingual learners. He argues that Children's L1 should be well developed to get their academic and linguistic performance of L2 maximized and Cummins emphasizes the use of L1 in initial education along with general social interaction in order to develop students' L2 proficiency. So that, Cummins "Developmental Interdependence Theory" suggests that growth in a second language is dependent upon a well-developed first language. He proposes through this hypothesis that "the level of L2 competence which a bilingual child attains is partially a function of the type of 
competence the child has developed in L1 at the time when intensive exposure to L2 begins" (Cummins, 1979).He goes on to suggest that the usage of certain functions of language and the development of L1 vocabulary and concepts are vigorously encouraged by the child's linguistic environment outside of school. He emphasizes the fact that when one is L1 is strongly developed "then intensive exposure to L2 is likely to result in high levels of L2 competence at no cost to L1 competence" (Cummins, 1979). At the same time he reminds the fact that had the students of less developed L1 skills been exposed to L2 in the initial grades they would have probably less progressed "the continued development of L1". This will negatively affect the development of L2, in turn. In brief, this hypothesis suggests that "there is an interaction between the language of instruction and the type of competence the child has developed in his L1 prior to school". This view reassures the need of paying relevant attention to one's L1 skills to lay down a strong basis for further language learning that may take place later. Cummins reviews the research evidence, which is related to the Developmental Interdependence Hypothesis and then deals with the "mechanisms" through which a child's L1 experience may influence the development of L2 skills. Yet, I focus on the latter which I find is more important to discuss here. Before I go into detail with Cummins' argument, I turn to highlight SkunabbKangas and Toukomaa's views quoted by Cummins to show the importance of mother tongue development in school subjects "which require abstract modes of thought" (Cummins, 1979). They view that the development of abstract thinking and conceptualization in L2 will be facilitated by initial mother tongue conceptualization and abstract thinking in the early stages. This shows the importance of mother tongue development in the early stage not only to promote L2 development but also to be excelled even in L1, an achievement at no cost to achievement in L2.

When the mechanisms which children's knowledge of L1 might interact with the language of instruction are focused, Cummins brings out the importance of students' "linguistic experience prior to school" which contributes to different "home-school language switch". In a situation Cummins suggests that L1 experiences of the students strongly promotes "fluent reading skills" with the help of "the vocabulary concept knowledge" and "the decontextualized knowledge" of the child and the extent to which the child has acquired "certain metalinguistic insights" in relation to the given text (Cummins, 1979). Further Cummins suggests that if the students have developed their conceptual knowledge in L1, they will encounter most of the concepts in L2 while reading and thereby students will be motivated to learn L2. Cummins further elaborates the point as follows: "The fact that the children are already familiar with the concepts encountered in learning L2 means that their task is essentially learning a new label to for an already existing concept"(Cummins, 1979). The article also talks about "An Interaction Model of Bilingual Education" and suggests that external factors like motivation, increasingly symbolic educational environment, students' interaction with the educational environment, attitudes towards L1 and L2 cultures and the amount of exposure to L2 and L2 speakers are also some determining factors, which characterize the competency of bilingual learners (Cummins, 1979). Thus, the success in Bilingual Education, according to Cummins is the balanced approach of the abovementioned three aspects. Isolating 'motivation' out of all those linguistic variables, Cummins suggests that "motivation to learn L2 is likely to be low when the learning of L2 is regarded as a threat to the children's identity". This is an important point to discuss further in terms of bilingual learners because they feel less 'forced' to learn L2 when compared to L2 learners in monolingual mode. Cummins' "Threshold Hypothesis" suggests, "A child must attain a certain level of proficiency in both the native and second language in order for the beneficial aspects of bilingualism" to accumulate. A number of Theoretical Aspects and Research Findings related to Bilingual Education have been brought forward by Cummins to prove the above fact and especially he quotes Lambert and Tucker's (1972) suggestion as follows: “... the bilingual child engages in a form of "contrastive linguistics" by comparing similarities and differences in the vocabulary and syntactic structures of his two languages" (Cummins, 1979).

To engage in "contrastive linguistics", a student should have the proficiency of both the native and second language. Thus, those who do not have a chance to master their L1 at the beginning, probably during their primary education, may come up with difficulties in learning a L2 in a later stage. So that the interaction with the environment through underdeveloped L1 or L2 in terms of both input and output at the beginning, may cause to become poor in language proficiency later. At this point Cummins comes out with two types of threshold levels as "lower threshold level of bilingual competence" and "higher threshold level of bilingual competence". The "lower threshold level of bilingual competence" avoids negative cognitive effects yet a "higher threshold level of bilingual competence" is "necessary to lead to accelerated cognitive growth" 
(Cummins, 1979). Further, he points out that the lower threshold level of bilingual competence leads bilingual children's competence in a language to be adequately weakened. However, he affirms further, the quality of a children's interaction with their educational environment, may be vary due to "the children's stage of cognitive development and the academic demands of different stages of schooling" (Cummins, 1979). At the same time he points out during the early grades students' interaction with environment and gradual cognitive development are less dependent on "the mediation of language" than at later grades. Thus, the students gain L2 skills from "an increasingly symbolic environment". Hence, Cummins states:"Thus, in the early grades the lower threshold may involve only a relatively low level of listening comprehension and expressive skills, but- as the curriculum content becomes more symbolic and requires more abstract formal operational thought processes - the children's "surface" L2 competence must be translated into deeper levels of "cognitive competence" in the language" (Cummins, 1979).

So that Cummins, citing Olson (1977), shows the importance of developing adequate literacy skills in the early stages because then only the students can absorb most types of subject matter content in the later grades. Olson suggests that "the acquisition of literacy skills" at the early grades "has more general cognitive significance" and it leads the students to become "proficient" in using "the logical or ideational functions of language" later on (Cummins, 1979). So that, as discussed above, it is important to develop necessary literacy skills (in L1 or L2) at the beginning, in order to excel in linguistic development of a child in the later stages. He goes on to suggest with that to attain a higher threshold level, it is important to maintain one's L1 skills (Cummins, 1979). Apart from the above theories discussed so far, Cummins is best known for his distinction between "Basic Interpersonal Communication Skills (BICS) and Cognitive Academic Language Proficiency (CALP). BICS or everyday conversational skills are quickly acquired, whereas CALP, the highly decontextualized, abstract language skills used in classrooms, may take 5-7 years or more to acquire. The essay, "BICS and CALP: Origins and Rationale for the Distinction" by Cummins states that the BICS/CALP distinction was not proposed as an overall theory of language but as "a very specific conceptual distinction that has important implications for policy and practice" (Cummins, 1979).To distinguish conversational fluency and academic aspects of L2 performance, Cummins (1980a) has done a reanalysis of large-scale language acquisition data from the Toronto Board of Education. When this particular observation is applied to Sri Lankan Bilingual Education program, it is obvious that those students, too, are quite comfortable in BICS rather than CALP. This observation based on the data collection will be discussed in detail in the analysis. Cummins highlights the importance of distinction between BICS and CALP because students may come to a conclusion and may 'prematurely exit' to "English - only" programs based on their "surface-level fluency", a linguistic façade. Thus, BICS cannot be used as a measurement of actual academic performance without evaluating one's CALP.

\section{Methodology}

The methodology used in this study is taken from the discipline of Sociolinguistics. The basic techniques were the distribution of questionnaires, which were followed up with interviews with the responders. Although obtaining such linguistic data is commonplace in Linguistics, it was not as smooth in practice as in theory, possibly because of the sensitive nature of the subject matter; that is, the prestige accorded to English in bilingual mode. The procedure involved was that the questionnaires were distributed personally and randomly to selected sixty students in grade nine, in four schools in Ratnapura district. Three of those schools were National Schools (R/Ferguson High School, R/Sumana Balika Vidyalaya and R/Seevali Central College) while one was a Navodya School (R/Dharmapala Maha Vidyalaya). Another twelve questionnaires were given to teachers who work in the Bilingual Program (both English teachers and non- English teachers) while eight questionnaires were given to teachers who work in the primary education. Informants (teachers) were subsequently interviewed by appointment two days after, to clarify comments made on the questionnaire and to elicit further information, such as the informants' personal opinions on speaking difficulty faced by bilingual learners. There were several drawbacks too. They were initially the practical difficulties. However, those drawbacks were to be anticipated and due to the tediousness of the proceedings, two days were sacrificed per one school. Such problems of practicality however, are mostly woven into the methodology and cannot be avoided. As stated earlier, the data for the study consist of answers to a questionnaire and the responses on an oral interview with the informants (only with the teachers). The sample size was 60 with grade nine students of Bilingual Program randomly selected from 4 schools and 12 teachers including English 
teachers, non- English teachers who work in the Bilingual Program and 8 primary teachers also randomly selected from the same 4 schools. I did not have a choice in terms of schools because there were only 4 schools with grade 9 Bilingual Program in Ratnapura District. There were about four more schools with Bilingual Program but they did not have particularly grade 9 bilingual classes since it was newly implemented in those schools. I planned to get 15 students from each school but the Navodya School (R/Dharmapala Maha Vidyalaya ) had only 5 students in the grade 9 Bilingual Program and unfortunately one was absent on that day. Thus, the rest of the questionnaires (11) were divided among the other three schools, so that 20 were taken from R/Ferguson High School, 18 from R/Sumana Balika Vidyalaya and another 18 from R/Seevali Central College. Wherever possible, I attempted to have a well-balanced sample taking both girls, boys but again I was limited in my choice, and in reality, it turned out to be somewhat sexist in that because two of the schools were girls' schools. Of the total, 16 were boys (2 from Dharmapala Vidyalaya and 14 from Seevali Central College) while the rest - 54 happened to be girls.

\section{Results and Discussion}

Tracing the history of Bilingual Education, anyone can observe the prevailed attitudes on this particular issue in the past. Those attitudes were initially negative. Many people believed that Bilingual Education might cause problems because in situations where children are virtually cut off from their family language and 'submerged' in a second language for long periods they "may begin to lose the family language before they have developed an age-appropriate mastery of the new language". (Lightbown and Spada, 1999, p.3) Some research findings, too, have aggravated the situation. For instance, Darcy, 1953, Pearl, and Lambert, 1962 viewed Bilingual Education as a cause of "mental confusion" and something that may cause "language handicaps". Apart from them, the "linguistic mismatch hypothesis" which suggested that mismatch between the language of the home and the language of the school leads to academic retardation (Downing 77-88) also brought up the negative attitudes on bilingual education. On contrary to all these 'negative' views, by now, researchers have identified the 'positive' aspects of Bilingual education. For instance, a large number of recent studies done by Baker (2000), Cummins (2000) and Kangas (2000) have shown that Bilingualism can positively influence both cognitive and linguistic development. Therefore, I will explore these theories accordingly, along with my findings as the discussion goes on. Cummins emphasizes in his article "Linguistic Interdependence and the Educational Development of Bilingual Children", that the well-developed L1 "is unaffected by intensive exposure to L2" (Cummins, 1979). In other words, tendency to occur "subtractive bilingualism" is minimal when L1 is well developed at the early stages of life. In addition, Cummins' Developmental Interdependence Hypothesis suggests the growth in a second language is dependent upon a well-developed first language. This view is quite different from the axiomatic: a child should be exposed to a language as early as possible in order to achieve competency in second language learning. In the past, everyone was interested on switching into an English only mode to master the global language - English. Yet, Cummins' suggestion that to achieve competency in L2, one should first master his or her L1 well seems revolutionary.

When I turn to the collected data, I can see that the majority of the sample population (55 out of 60) have gained their primary education in Sinhala medium - (L1). Further, 53\% of the sample population has passed their grade five-scholarship examination, which is considered a high standard language (L1) proficiency test in Sri Lanka. Even those students, who have not passed their grade five-scholarship examination, have scored more than 120 marks, which is not easy for someone who is not good at his or her L1. Both these facts ensure the point that these focused students have already mastered their L1 into an accepted standard yet they find difficult in speaking in English in terms of their L2. Thus, my findings so far, do not consist with Cummins' Developmental Interdependence Hypothesis. I will explore them further as my discussion proceeds. "Proposals of the National Education Commission - Sri Lanka", states under Bilingual Education Policy - 2003, that "students will reach an acceptable level of proficiency in English at the end of junior secondary education without jettisoning Sinhala and Tamil which will continue to be the medium of instruction in selected subjects."At this moment, before I proceed in my discussion further, again I turn to the collected data to elicit the problematic situation. Collected data clearly shows that the majority of students (39 out of 60) come up with the idea that 'speaking' is the most difficult skill to master. Thus, it shows, though the policy makers expect students to achieve proficiency when they come to grade nine after spending three years in the Bilingual Program, they are not competent enough in their L2 speaking and students find more difficult to 
deal with the academic language rather than the everyday conversational language. This particular issue can be evaluated with the theory discussed in Literature Review, to find out the reasons behind this drawback. Cummins, who has extensively researched on second language learning, comes up with the distinction between BICS and CALP. With this hypothesis, he suggests that BICS or everyday conversational skills are quickly acquired, whereas CALP or the highly decontextualized, abstract language skills used in classrooms may take 5-7 years or more to acquire. This particular hypothesis is very important when addressing the lack of English speaking skills in grade nine bilingual learners because, grade nine students have spent only three years in the bilingual program, after a long period of monolingual education. Data show that the majority of students are quite comfortable with their everyday language and it may be because they catch up the conversational language within 2-3 years by the time they come to grade nine. On the other hand, as Cummins points out students find more difficult in academic language, may be because still they are 'on the way to master' CALP since it takes at least 5-7 years to achieve competency. At the same time, $86 \%$ of students have assured the need of specialized vocabulary when they learn subject matter in English. This also shows their difficulty in mastering Cognitive and Academic Proficiency as Cummins points out in his hypothesis.

At this point, I would like to discuss Cummins' "Threshold Hypothesis" which suggests that a child must attain a certain level of proficiency in both the native and second language to bring forward the maximum beneficial aspects of Bilingualism. He further emphasizes the importance of bilingual learners' social and linguistic experiences prior to their schooling, because it results with "a higher level of threshold" which ultimately facilitates cognitive and academic proficiency in L2 that will take place in later stages. This is mainly because due to the conceptualization take place in L1 which could be 'transferred' into L2 when need arises. When I apply this view into my findings, it is clear why all the teachers in the sample population come up with the same idea that those students who are good at their mother tongue are equally good at their L2 as well. Cummins elaborates this particular idea in terms of reading. When students have already armed with conceptualization in their L1, it would facilitate their L2 acquisition. Yet, this view can be further discussed even in terms of speaking of bilingual learners because as Roger Browns suggests, "the presence of the concept is an invitation to acquire the word" (Cummins, 1979). So that if the teachers in Bilingual Program encourage learners to bring forward L1 experiences to bilingual classroom while teachers connect their particular subject matters with students L1 experiences, bilingual learners may 'catch up' the relevant specific vocabulary they need. In this occasion, common strategies found in bilingual classrooms like "codemixing" and "code- switching" may be useful. The following example extracted from "Bilingual Education Teacher Development Manual" published by NIE, Sri Lanka, elicits my point further. For example, "when we eat rice it goes through the "Annasrothaya" and reaches the "Amashaya". To this, the teacher wrote, "when we eat rice it goes through the oesophagus and reaches the stomach". She made us to repeat this several times. As result, we became familiar with the words" (Bilingual Education, Teacher Developmental Manual, 2007, p.60). In addition, it is important to look at Bowen and Tucker's, Paulson, and Fishman's view on Bilingual Education, which suggests that Bilingual Education is a collection of "societal factors" rather than "independent variables". So that the extent which students' interaction takes place with their immediate environment is also a decisive factor in analyzing the difficulties of speaking in English, find in Bilingual Program.

Discussing "An Interaction Model of Bilingual Education", Cummins suggests that "motivation to learn L2 is likely to be low when the learning of L2 is regarded as a threat to the children's identity". This is an important point, which is declared by Cummins in terms of minority learners, but I hope to discuss this view in terms of Sri Lankan society, based on my collected data. A $66 \%$ of sample population believes that they feel uncomfortable in using L2 freely because they think that they will be ridiculed by others when they make mistakes in L2. This view is quite surprising sometimes because many believe that bilingual learners feel less 'forced' to learn L2 and quite comfortable in using L2 when compared to L2 learners in monolingual mode. Yet this data go on to show the 'prejudices' find in our society, which might negatively affect L2 learning. Thus, it is important to create a stress free and learner friendly environment to improve their L2 usage even in Bilingual Education Program where students can be encouraged to answer questions, express themselves when cognitive/academic teaching- learning process takes place. However, Cummins points out the importance of maintaining a higher threshold level by exposing the child extensively to his or her L1 at the 
early childhood in order to develop successful second language skills in the later stages and emphasizes the importance of mastering a language as early as possible, to be successful in language acquisition.

\section{Conclusion \& Recommendations}

This research was undertaken in order to examine the difficulties in speaking in English faced by students in the Bilingual Education Program in selected schools in Ratnapura district. The introduction shows the basis on which the hypothesis was created, where it was proved that it was the difficulties in speaking faced by students in the Bilingual Program that act as a barrier in achieving academic performances. Students as well as teachers of the sample population, admit the fact that there is a difficulty in dealing with cognitive/academic language. Further, when collected data is analyzed and discussed along with the theory, I find the importance of L1 competency in developing L2, at the early stages of learners. This view is extensively evaluated with the theories like "Developmental Interdependence Theory", "The Threshold Theory" and the "BICS and CALP Distinction" by Cummins. When I evaluated collected data along with the theory, I find that the students of my sample population have mastered their L1 up to a certain positive level, yet they come up with difficulties in speaking in English. However, the collected data further elicit the difficulty of speaking in English that students face with. It is the academic language that they find difficulty to deal with, mainly because it takes time to master CALP rather than BICS. At the same time factors like the amount of threshold level developed, may also promote the Cognitive and Academic Proficiency of students along with number of external factors like motivation. Thus, it can be concluded that promoting L2 learning in the secondary level more or less depends on developing one's L1 competency at the young age.

\section{References}

Baker, C. (2000). A parents and teachers guide to bilingualism. 2nd Edition. Clevedon, England: Multilingual Matters.

Bilingual Education. (2007). Teacher Developmental Manual. NIE, Maharagama, Sri Lanka, 2007.

Cummins, J. (1979). Linguistic Interdependence and the Educational Development of Bilingual Children. Review of Educational Research, 49, 222-251.

Cummins, J. (1980). The Cross-Lingual Dimensions of Language Proficiency: Implications for Bilingual Education and the Optimal Age Issue. TESOL Quarterly, 14(2), 175-187.

Darcy. N. T. (1953). Bilingualism and the measurement of intelligence: Review of a decade of research. Journal of Genetic Psychology, 103, 259-282.

Downing, J. (1979). Reading and reasoning. New York, NY: Springer-Verlag, 77-88

Lightbown, P. M. \& Spada, N. (1999). How Languages are Learned, New York: Oxford University Press

Olson, D. R. (1977). From utterance to text: The bias of language in speech and writing. Harvard Educational Review, 47, 257-281.

Peal, E. \& Lambert, M. (1962).The relation of bilingualism to intelligence. Psychological Monogruph, 76(546), 1-23.

Skutnabb-Kangas, T. \& Toukomaa, P. (1976). Teaching migrant children's mother tongue and learning the language of the host country in the context of the sociocultural situation of the migrant family. Helsinki: The Finnish National Commission for UNESCO.

Skutnabb-Kangas, T. (2000). Linguistic genocide in education-or worldwide diversity and human rights? Mahwah, NJ: Lawrence Erlbaum Associates. 